\title{
Spectral analysis of EEG signals among people with different levels of personal anxiety
}

\author{
Svitlana Shvaiko', Olena Dmytrotsa', Tetiana Poruchynska', Andrij Poruchynskyi', \\ Oleksandr Zhuravlov' ${ }^{1}$, Alevtyna Morenko'
}

'Lesya Ukrainka Eastern European National University

Shvaiko S, Dmytrotsa O, Poruchynska T, Poruchynskyi A, Zhuravlov O, Morenko A. The spectral analysis of eeg signals among people with different levels of personal anxiety. J Pre-Clin Clin Res. 2018; 12(1): 22-25. doi: 10.26444/jpccr/87143

\begin{abstract}
Introduction. The study of electrical activity peculiarities in the cerebral cortex among people with different levels of anxiety and the dependence of EEG rhythms from the level of personal anxiety (PA) is relevant and needs to be studied. As a result of our research, we have found that during the baseline EEG among people with a moderate level of personal anxiety, the intensity of alpha rhythm predominates in the posterior areas of the cerebral cortex. People with a high level of PA had the highest rates of beta rhythm intensity. An inverse relationship between EEG main rhythms and the level of anxiety is noted. Thus, people, who were involved in our study, with the moderate level of PA had the following results: the steadier the PA, the less the intensity of the PA level. People with a high level of PA had positive connections between the alpha and beta rhythms and level of anxiety, as well as a higher activity in the right-hemisphere of the cortex, which indicates the contribution of this hemisphere in the high level PA in studies.

Materials and method. The power of EEG rhythms among people (aged 18-22) with different levels of anxiety was studied. Registration of the cerebral cortex electrical activity was carried out using the «Neurocom».device. During the electroencephalogram recording, electrodes were placed on the international system 10/20. The spectral power values were analyzed in the standard physiological frequency bands: delta (1-4 Hz), theta (4-8 Hz), alpha (8-14 Hz), beta (14-35 Hz). For the spectral EEG characteristics, an absolute $(A C P, \mu \mathrm{V})$ and relative spectral power $(\mathrm{RSP}, \%)$ were used. The analysis of power indices was carried out in a state of rest with closed eyes (baseline), and a state of rest with open eyes during task solving as an emotional load.

Results. We have found that during the EEG baseline people with the moderate level of PA had high indexes of alpha rhythm in the posterior areas of the cerebral cortex. Eyes' opening caused an increase of the intensity of theta rhythm in the frontal area of the cerebral cortex. During the intellectual task conducting, we noticed that the increase of intensity of beta rhythm in the frontal, temporal and end-lobe parts, whereas in the central and vertex parts of the cortex the intensity of theta rhythm predominated. Among people with a high level of PA during the baseline probe, the highest rates of beta rhythm intensity in the posterior areas of the cerebral cortex were fixed. In the state of rest with open eyes, the activity of theta rhythm was revealed. The alpha rhythm had the highest rates of intensity in the posterior area of the right hemisphere. During the intellectual task, the intensity of beta rhythm predominated. In the frontal and central parts of the cortex, the intensity of theta rhythm predominated.

Conclusions. During the baseline probe among people with a moderate level of PA, the intensity of alpha rhythm in the posterior areas of the cerebral cortex predominated, among people with a high level of PA - beta rhythm. eyes open caused an increase in the intensity of theta rhythm in the frontal area of the cerebral cortex of people with a moderate level of PA, and alpha rhythm in the posterior area of the right hemisphere. During the intellectual task conducted among people with a moderate level of PA, we noticed an increase in beta rhythm intensity in the frontal, temporal and end-lobe parts, but in the central and vertex parts of the cortex the intensity of theta rhythm predominated. While in another group of people who were involved in study, the intensity of beta rhythm predominated; in the frontal and central parts - theta rhythm. With a moderate level of PA, there were significant negative links between the level of anxiety and intensity of alpha, beta, theta rhythms. With a high level of PA, positive links between the intensity of alpha and beta rhythms and the level of anxiety were noticed; the intensity of EEG rhythms in this group of examined people was correlated with the right parts of the brain, which indicates to a big contribution of this part in the high level of PA among the examined people.
\end{abstract}

\section{Key words}

personal anxiety, encephalogram, alpha, beta, delta, theta rhythms, spectral intensity of rhythm

\section{INTRODUCTION}

Currently, the problem of anxiety is becoming more urgent. This is connected with changes in the social sphere, stressful pace of life, and other factors. Anxiety, as a personality trait, is associated with genetically-determined properties

Address for correspondence: Olena Dmytrotsa, Lesia Ukrainka East European National University, Nikishewa 14, 43010 Lutsk, Ukraine

E-mail:dmytrotsa2017@ukr.net

Received: 3 December 2017; accepted: 22 March 2018 of the human brain, which determine the increased level of emotional excitement. Anxiety is inseparable from the concept of human life, its organizing role as a factor of mental processes integration is undeniable, and its destabilizing role is also obvious [1].

Current studies of anxiety aim at distinguishing situational anxiety associated with a particular external situation, and personal anxiety, which is a stable personality trait, as well as the development of methods for anxiety analysis [3]. Personal anxiety refers to stable individual characteristics, 
reflecting the ability of the subject to anxiety and involves the presence of a tendency to accept a broad range of situations as being threatening, answering each of them with a certain reaction [2].

The issue on the connection of alpha-rhythm intensity with the personal anxiety level has not been clarified. Modern scientific sources testify to the constant connection between the development of the alpha-rhythm and personal anxiety level. It has been established that healthy participants had alpha-rhythm capacity in a relative state of rest. The alpharhythm capacity positively correlated with the personal anxiety level by experimental task conducting. It was also discovered that participants with high levels of personal anxiety were different from individuals with moderate level of personal anxiety because of the high level of alpha-rhythm in the front parts of both hemispheres and in the vertex of the left hemisphere. The presence of significant negative connections between alpha rhythm capacity and the level of personal anxiety indicates that the individual factors of the alpha-rhythm capacity is stable and insensitive to the situational influences in terms of relative state of rest [3]. Therefore, the study of the features of cerebral cortex electrical activity of people with different levels of anxiety and the establishment of the dependence of EEG rhythms on the level of personal anxiety is relevant.

\section{MATERIALS AND METHOD}

In the study of EEG rhythm capacity, participants (aged 18-22 years) were divided into 2 groups (10 people) by level of personal anxiety (PA): Group I - surveyed with moderate level of PA, group II - surveyed with high level of PA. Investigations of the level of anxiety were carried out according to the generally accepted method (SpielbergerHanin anxiety scale).

The questionnaire score sheet includes instructions and 40 questions, 20 of which are to assess the level of situational anxiety (ST) and 20 to assess the level of personal anxiety (PA). The research was conducted individually. The respondent were asked to answer a number of questions quickly, each of which had 4 answers by intensity. The answer should be individual. Determination of personal anxiety rates was carried out with the help of a key, given that the aggregate end result for each of the scales was in the range of $20-80$ points: up to 30 points - low, 31-44 - moderate, 45 and more - high.

The research was conducted in the Laboratory of Age Neurophysiology of the Department of Human and Animal Physiology of Lesya Ukrainka Eastern European National University.

Registration of cerebral cortex electrical activity was carried out using the "NeuroCom" device created by the scientific and technical centre of electronic medical devices and technologies "KhAI-Medica" of the National Aerospace University. During the recording of the electroencephalogram, the electrodes were placed in an international system of $10 / 20$ at 19 points on the scalp (Fp1, Fp2 - anterolateral, F3, F4 - posterior scapular, F7, F8 - lateral frontal, T3, T4 - frontline, C3, C4 - central; T5, T6 -posttemporal, P3, P4 - parietal, O1, O2 - occipital, Fz, Cz, Pz - sagittal frontal, central and parietal distances). The spectral capacity values were analyzed in the standard physiological frequency ranges: delta $(1-4 \mathrm{~Hz})$, theta $(4-8 \mathrm{~Hz})$, alpha $(8-14 \mathrm{~Hz})$, beta
(14-35 Hz). In the analysis of experimental data, we used the method of integral quantitative analysis of the EEG-pattern, which allowed evaluation of the effect of the the spectral capacity of the partial ranges of the EEG, and determination of the importance of the particular partial components of ECG for the formation of a single ECG pattern.

Rhythm index was determined; this in the presence of the particular rhythm (activity) related to the whole time of EEG registration, which is measured in percents. According to EEG of different people, alpha-rhythm fluctuates from $0-100 \%$. It should be set at $75-95 \%$, and in case it is lower than $50 \%$ it should be considered as pathology. A high alphaindex testified to the superiority of alpha-rhythm EEG, and low showed its mere superiority. Decrease in the alpha-index indicated negative EEG dynamics, which is related to the increase of the theta and delta indexes [1].

As the spectral characteristics of the EEG, an absolute spectral power (ASP, $\mathrm{MV}$ ) was used - an indicator of the amplitude signal characteristic; relative spectral power (RSP, $\%)$ - and characteristic of the spectrum structure (ratio of different EEG ranges).

The EEG rhythm capacity indices of male and female participants were not significantly different, which enabled us to consider the indicators for the whole group of participants. The analysis of capacity indexes was carried out in the following experimental situations: rest state with closed eyes (baseline), rest state with open eyes; solving the problem as an emotional load.

During the statistical data processing, determination of the correlation coefficient according to Spearman was carried out, for the purpose of the connection estimation between ASP of particular EEG rhythms and the level of personal anxiety. Depending on the correlation coefficient, the connections were divided into: strong (tight) correlation 0.7 $<\mathrm{r}<1$; reasonable correlation- $0.3<\mathrm{r}<0.5$; weak correlation $-0.2<\mathrm{r}<0.3$; absence of correlation $-\mathrm{r}<0.19$.

For further comparison of the indicators, Mann-Whitney $\mathrm{U}$ criterion with Bonferron's correction was used. The result was statistically important at a significance of $\mathrm{P}<0.05$.

The obtained results were processed by methods of variation statistics, taking into account parametric and nonparametric criteria comparing average values. We distinguished mean values of the indicators $(M)$, the mean error value $(m)$, Student's reliability criteria $(\mathrm{t})$ when comparing the average values, and the degree of probability ( $\mathrm{p})$. At $\mathrm{p} \leq 0.05 ; \mathrm{t} \geq 2.1$ the difference was considered as significant.

\section{RESULTS}

Taking into account the EEG capacity indexes among people with high and moderate levels of PA during baseline sample, it was found out that alpha-rhythm capacity dominated among people with a moderate level of PA. The increase in rhythm capacity was in the occipital cortex, mainly in the left hemisphere.

By assessing the beta-rhythm capacity indexes during the baseline sample, high indexes of ASP were specified among people with a high level of personal anxiety. The highest rates of beta-rhythm capacity were identified in the occipital cortex. Among people with a moderate level of PA the preference of beta-rhythm capacity was observed in the left anterio-frontal cortex. 
Taking into account the factors of theta-rhythm capacity, it was found that people with a moderate level of PA had ASP indexes in the frontal and occipital cortex. Among people with a high level of $\mathrm{PA}$, theta-rhythm capacity indexes were identified in the central and occipital cortex of the left hemisphere. In the dorsal temporal lobe, high capacity indexes were distinguished among students with a high level of PA.

In rest state with open eyes, the absolute value of the alpharhythm is prevailing among people with moderate level of PA.

The highest rates of alpha-rhythm capacity are distinguished in frontal cortex. People with high level of personal anxiety have alpha-rhythm capacity only in right central cortex C4. Students with high level of PA have high level of alpharhythm in occipital cortex. Participants with mean level of PA in rest state with open eyes had high indexes of beta-rhythm capacity in frontal and temporal lobe. The highest indexes of beta-rhythm were revealed among students with mean level of PA in Fp1. In central, vertex and temporal cortex the high level of beta-rhythm was distinguished among people with high level of PA.

Among people with a mean level of PA at the rest state with open eyes, high indexes of theta-rhythm capacity were distinguished in the frontal cortex. Students with a high level of PA had theta-rhythm capacity only in the left central cortex.

EEG registry during the task conducting allows identification of the higher indexes of ASP alpha-rhythm in the frontal and occipital cortex among people with a mean level of PA. The highest rates were observed for the alpharhythm capacity in Fp1, Fp2 rejections. Among people with a high level of PA, high indexes of alpha-rhythm capacity were revealed in the temporal, central and occipital cortex.

Among people with a mean level of PA, high indexes of beta-rhythm in Fp1, Fp2, T3, T5, T6, O2 were distinguished. In the occipital and central cortex, the highest indexes of beta-rhythm capacity were revealed among students with a high level of PA. In assessing the theta-rhythm, it was found that indexes obtained during EEG registry among people with a moderate level of PA, prevailed. The highest rates of theta-rhythm capacity were revealed in the frontal, and the least - in the temporal cortex.

Taking into account the factors of the relative EEG capacity, it was found that among people with a moderate level of PA, alpha-activity prevailed (Fig. 1.), and that people with a high level of PA had a high beta-rhythm (Fig. 2). However, the theta-rhythm index showed an unusual difference among the students with different levels of PA.

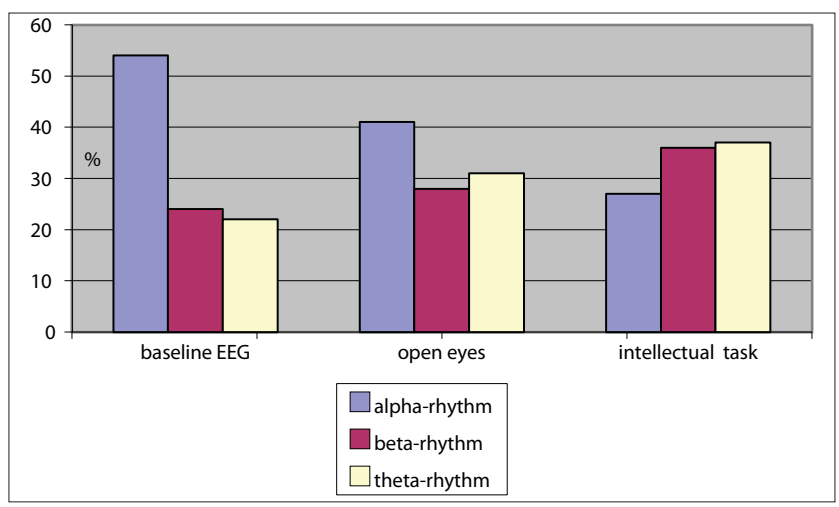

Figure 1. Distribution of specific EEG capacity rhythms among people with moderate level of PA in different conditions of stimulation
In the rest state with open eyes, students with moderate level of PA and people with high level of PA have thetaactivity domineering. The least in spectral range was specific part of beta-activity among people with different level of PA (see Fig. 1, 2).

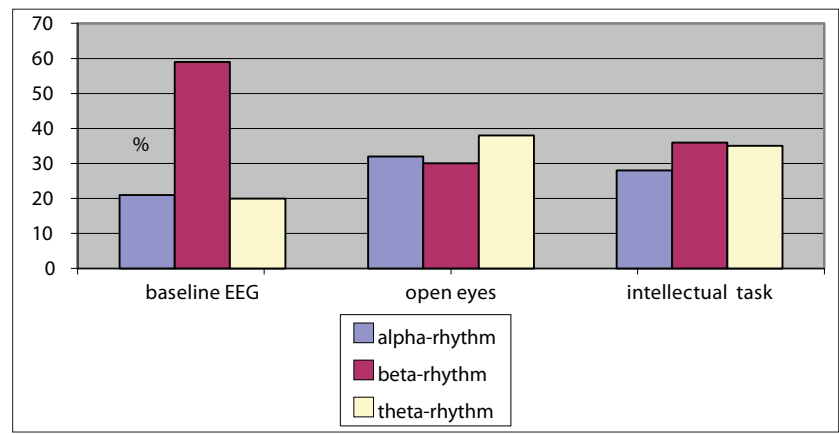

Figure 2. Distribution of specific EEG capacity rhythms among people with high PA under different stimulation conditions

Conducting a mental task with an emotional load by the students with a mean level of PA, bioelectrical activity of the cortex was characterized by the dominance of thetaactivity, Among people with a high level of PA, the specific beta-rhythm part in the overall spectral range alpha-activity part, prevailed among students with a moderate level of PA (Fig. 1, 2)

\section{DISCUSSION}

The obtained results were found in the publications by VB Pavlenko and CV Chornyi $[4,5]$ who, in their studies, also found significant connections between the beta-rhythm capacity and high level of PA. Since the beta-rhythm is regarded as a marker of psychological stress, we can predicate mnestic activity strengthening among people with a high level of anxiety. In the rest state with closed eyes, particular negative connections between all rhythms capacity and mean level of personal anxiety were observed. This may be connected with that fact that the more stable the personal anxiety index, the less the EEG rhythm capacity. The question about the connection between alpha-rhythm capacity and level of personal anxiety remains unclarified because of studies demonstrating an inverse relationship between alpharhythm capacity and level of personal anxiety. It was shown that among healthy participants [6], alpha-rhythm capacity, in terms of relative rest and conducting the experimental task, correlated positively with the level of personal anxiety. In another study [5] it was found that participants with a high level of personal anxiety were different from participants with a mean level of PA by a higher alpha-rhythm capacity inthe frontal cortex of both hemispheres and occipital cortex of the left hemisphere. The presence of negative connections between alpha-rhythm capacity and level of PA is explained by the fact that individual indexes of alpha-rhythm capacity are relatively stable, and less sensitive to the situational influences in terms of relative state of rest [4]. 


\section{CONCLUSIONS}

1. People with a mean level of personal anxiety during the baseline test had alpha-rhythm capacity in the posteriorassociation cortex. Eyes open caused an increase in thetarhythm capacity in the frontal cortex. While conducting mental tasks, an increase in beta-rhythm capacity was revealed in the frontal, temporal and occipital cortex, and in the central and vertical cortex, the theta-rhythm prevailrf.

2. Among people with a high level of $\mathrm{PA}$, during the baseline test the highest indexes of capacity were observed in betarhythm in the posterior-association cortex. At the rest state with open eyes, theta-rhythm activity was observed to be more generalized. Alpha-rhythm had the highest indexes of capacity in the posterior-association cortex of the right hemisphere. In conducting the mental task, the beta-rhythm capacity prevailed. In the frontal and central cortex, theta-rhythm capacity dominated.

3. Participants with a mean level of PA had negative correlations between level of anxiety and alpha-, beta, theta-rhythms. This indicates an inverse relationship between the above-mentioned rhythms and the level of anxiety: the more stable the personal anxiety, the less the EEG rhythm capacity. People with a high level of PA had positive correlations between alpha-, beta-rhythms and level of anxiety. Among people with a high level of PA,
EEG rhythm capacity correlated with the cortex of the right hemisphere, which indicated the great impact of this hemisphere on the high level of PA of the participants.

\section{REFERENCES}

1. Aftanas LI, Reva NV, Savotina LN. Neyrofiziologicheckie korrelyaty vyizvannih diskretnyih emotsiy u cheloveka: individualnyi analiz [Neurophysiologic controls of the evoked discrete emotions of person: individual analysis]. Ross. fiziol. zhurnal. 2004; 12: 1457-1471.

2. Aftanas LI, Reva NV, Varlamov AA. Analiz vyzvannoy sinhronizatsii i desinhronizatsii EEG pri emotsionalnoy aktivatsii cheloveka: vremennyie i topograficheckie harakteristiki [The analysis of the induced synchronization and desynchronization of the EEG with the emotional activation of the person: the temporary and topographic characteristics]. Zhurnal VND im. I. P. Pavlova. 2003; 4: 485-494.

3. Gordeev SA. Osobennosti bioelektricheskoy aktivnosti mozga pri vysokom urovne trevozhnocti cheloveka [The peculiarities of the bioelectrical activity of the brain with a high level of the human's alertness]. Fiziologiya cheloveka. 2007; 4: 11-17.

4.Pavlenko VB, Chernyi SV, Gubkina DG. EEG-korelyatyi trevogi, trevozhnocti i emotsionalnoy stabilnosti u vzroclyih zdorovyih icpytuemyh [EEG-equivalents of anxiety, alertness and emotional stability of healthy adults examinees]. Neyrofiziologiya. 2009; 5: 400408.

5. Knyazev GG, Savostyanov AN, Levin EA. Anxiety and synchrony of alpha oscillations. Int J Psychophysiol. 2005; 3: 175-180.

6. Balconi M. Brain oscillations and BIS/BAS (behavioral inhibition/ activation system) effects on processing masked emotional cues. ERS/ ERD and coherence measures of alpha band [Text] / M Balconi, G Mazza // Int J Psychophysiology. 2009; 158-165. 